\title{
Generalized Solution Concepts in Games with Possibly Unaware Players
}

\author{
Leandro C. Rêgo \\ Statistics Department \\ Federal University of Pernambuco \\ Recife-PE, Brazil \\ e-mail: leandro@de.ufpe.br
}

\author{
Joseph Y. Halpern \\ Computer Science Department \\ Cornell University \\ Ithaca-NY, U.S.A. \\ e-mail: halpern@cs.cornell.edu
}

\begin{abstract}
Most work in game theory assumes that players are perfect reasoners and have common knowledge of all significant aspects of the game. In earlier work [Halpern and Rêgo 2006], we proposed a framework for representing and analyzing games with possibly unaware players, and suggested a generalization of Nash equilibrium appropriate for games with unaware players that we called generalized Nash equilibrium. Here, we use this framework to analyze other solution concepts, with a focus on sequential equilibrium. We also provide some insight into the notion of generalized Nash equilibrium by proving that it is closely related to the notion of rationalizability when we restrict the analysis to games in normal form and no unawareness is involved.
\end{abstract}

\section{INTRODUCTION}

Game theory has proved to be a useful tool in the modeling and analysis of many phenomena involving interaction between multiple agents. However, standard models used in game theory implicitly assume that agents are perfect reasoners and have common knowledge of all significant aspects of the game. There are many situations where these assumptions are not reasonable. In large games, agents may not be aware of the other players in the game or all the moves a player can make. Recently, we [Halpern and Rêgo 2006] proposed a way of modeling such games. A key feature of this approach is the use of an augmented game, which represents what players are aware of at each node of an extensive form representation of a game. Since the game is no longer assumed to be common knowledge, each augmented game represents the game a player considers possible in some situation and describes how he believes each other player's awareness level changes over time, where intuitively the awareness level of a player is the set of histories of the game that the player is aware of.
In games with possibly unaware players, standard solution concepts cannot be applied. For example, in a standard game a strategy profile is a Nash equilibrium if each agent's strategy is a best response to the other agents' strategies, so each agent $i$ would continue playing his strategy even if $i$ knew what strategies the other agents were using. In the presence of unawareness this no longer make sense, since the strategies used by other players may involve moves $i$ is unaware of. We proposed a generalization of Nash equilibrium consisting of a collection of strategies, one for each pair $\left(i, \Gamma^{\prime}\right)$, where $\Gamma^{\prime}$ is a game that agent $i$ considers to be the true game in some situation. Intuitively, the strategy for a player $i$ at $\Gamma^{\prime}$ is the strategy $i$ would play in situations where $i$ believes that the true game is $\Gamma^{\prime}$. Roughly speaking, a generalized strategy profile $\vec{\sigma}$, which includes a strategy $\sigma_{i, \Gamma^{\prime}}$ for each pair $\left(i, \Gamma^{\prime}\right)$, is a generalized Nash equilibrium if $\sigma_{i, \Gamma^{\prime}}$ is a best response for player $i$ if the true game is $\Gamma^{\prime}$, given the strategies being used by the other players in $\Gamma^{\prime}$. We showed that every game with awareness has a generalized Nash equilibrium by associating a game with awareness with a standard game (where agents are aware of all moves) and proving that there is a one-toone correspondence between the generalized Nash equilibria of the game with awareness and the Nash equilibria of the standard game.

Some Nash equilibria seem unreasonable. For example, consider the game shown in Figure 1. One Nash equili-

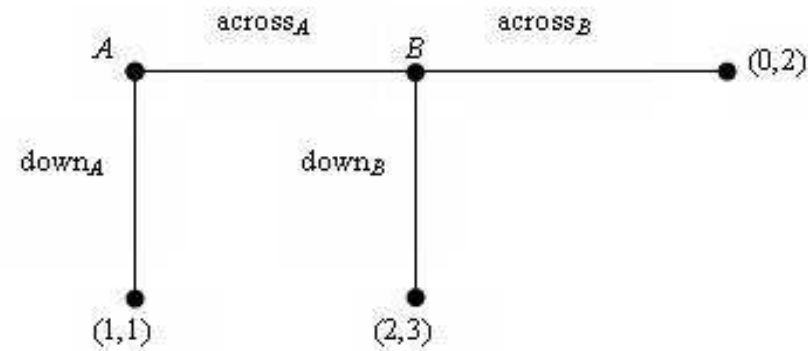

Figure 1: A simple game. 
brium of this game has $A$ playing down $A$ and $B$ playing $\operatorname{across}_{B}$. Since player $A$ chooses down $A$, player $B$ never gets the chance to move so whatever he does he will get the same payoff of 1 . However, if player $B$ is rational and ever gets to move, he will not choose move across ${ }_{B}$ since it gives a lower payoff for him than choosing move down $n_{B}$.

There have been a number of variants of Nash equilibrium proposed in the literature, such as perfect equilibrium [Selten 1975], proper equilibrium [Myerson 1978], sequential equilibrium [Kreps and Wilson 1982], and rationalizability [Bernheim 1984; Pearce 1984], to name just a few. Each of these solution concepts involves some notion of best response. Our framework allows for straightforward generalizations of all these solution concepts. As in our treatment of Nash equilibrium, if $\Gamma_{1} \neq \Gamma_{2}$, we treat player $i$ who considers the true game to be $\Gamma_{1}$ to be a different agent from the version of player $i$ who considers $\Gamma_{2}$ to be the true game. Each version of player $i$ best responds (in the sense appropriate for that solution concept) given his view of the game. In standard games, it has been shown that, in each game, there is a strategy profile satisfying that solution concept. Showing that an analogous result holds in games with awareness can be nontrivial. Instead of going through the process of generalizing every solution concept, we focus here on sequential equilibrium since (a) it is one of the best-known solution concepts for extensive games, (b) the proof that a generalized sequential equilibrium exists suggests an interesting generalization of sequential equilibrium for standard games, and (c) the techniques used to prove its existence in games with awareness may generalize to other solution concepts.

We also provide some insight into the notion of generalized Nash equilibrium by proving that, in a precise sense, it is closely related to the notion of rationalizability when we restrict the analysis to games in normal form and no unawareness is involved (although the underlying game is no longer common knowledge among the players). Roughly speaking, a normal form game can be thought as a one-shot extensive game where no player knows the move the others made before they make their own move. Intuitively, in standard games, a strategy is rationalizable for a player if it is a best response to some reasonable beliefs he might have about the strategies being played by other players, and a strategy is part of a Nash equilibrium if it is a best response to the strategies actually played by the other players. Since, in games with awareness, the game is not common knowledge, a local strategy for player $i$ in $\Gamma^{+}$is part of a generalized Nash equilibrium if it is a best response to the strategies played by the opponents of player $i$ in the games player $i$ believes his opponents consider to be the actual one while moving in $\Gamma^{+}$. Note that the line between rationalizabilty and generalized Nash equilibrium is not sharp. In fact, we are essentially able to prove that a strategy is rationalizable in a standard game $\Gamma$ iff it is part of generalized
Nash equilibrium of an appropriate game with awareness whose underlying game is $\Gamma$.

The rest of this paper is organized as follows. In Section 2, we give the reader the necessary background to understand this paper by reviewing our model for games with awareness. In Section 3, we review the definition of sequential equilibrium for standard games and define its generalization for games with awareness. In Section 4, we define the concept of conditional sequential equilibrium for standard games, and prove that there is a one-to-one correspondence between the generalized sequential equilibria of a game with awareness and the conditional sequential equilibria of the standard game associated with it. In Section 5, we analyze the connection between rationalizability and generalized Nash equilibrium. We conclude with some discussion of solution concepts in games with unaware players in Section 6.

\section{GAMES WITH AWARENESS}

In this section, we introduce some notation and give some intuition regarding games with awareness. We encourage the reader to consult our earlier paper for details.

Games with awareness are modeled using augmented games. Given a standard extensive-form game described by a game tree $\Gamma$, an augmented game $\Gamma^{+}$based on $\Gamma$ augments $\Gamma$ by describing each agent's awareness level at each node, where player $i$ 's awareness level at a node $h$ is essentially the set of runs (complete histories) in $\Gamma$ that $i$ is aware of at node $h$. A player's awareness level may change over time, as the player becomes aware of more moves.

Formally, a (finite) extensive game is a tuple $\Gamma=$ $\left(N, M, H, P, f_{c},\left\{\mathcal{I}_{i}: i \in N\right\},\left\{u_{i}: i \in N\right\}\right)$, where

- $N$ is a finite set of players.

- $M$ is a finite set whose elements are the moves (or actions) available to players (and nature) during the game.

- $H$ is a finite set of finite sequences of moves (elements of $M$ ) that is closed under prefixes, so that if $h \in H$ and $h^{\prime}$ is a prefix of $h$, then $h^{\prime} \in H$. Intuitively, each member of $H$ is a history. We can identify the nodes in a game tree with the histories in $H$. Each node $n$ is characterized by the sequence of moves needed to reach $n$. A run in $H$ is a terminal history, one that is not a strict prefix of any other history in $H$. Let $Z$ denote the set of runs of $H$. Let $M_{h}=\{m \in M: h$. $\langle m\rangle \in H\}$ (where we use - to denote concatenation of sequences); $M_{h}$ is the set of moves that can be made after history $h$.

- $P:(H-Z) \rightarrow N \cup\{c\}$ is a function that assigns to each nonterminal history $h$ a member of $N \cup\{c\}$. (We 
can think of $c$ as representing nature.) If $P(h)=i$, then player $i$ moves after history $h$; if $P(h)=c$, then nature moves after $h$. Let $H_{i}=\{h: P(h)=i\}$ be the set of all histories after which player $i$ moves.

- $f_{c}$ is a function that associates with every history for which $P(h)=c$ a probability measure $f_{c}(\cdot \mid h)$ on $M_{h}$. Intuitively, $f_{c}(\cdot \mid h)$ describes the probability of nature's moves once history $h$ is reached.

- $\mathcal{I}_{i}$ is a partition of $H_{i}$ with the property that if $h$ and $h^{\prime}$ are in the same cell of the partition then $M_{h}=M_{h^{\prime}}$, i.e., the same set of moves is available for every history in a cell of the partition. Intuitively, if $h$ and $h^{\prime}$ are in the same cell of $\mathcal{I}_{i}$, then $h$ and $h^{\prime}$ are indistinguishable from $i$ 's point of view; $i$ considers history $h^{\prime}$ possible if the actual history is $h$, and vice versa. A cell $I \in \mathcal{I}_{i}$ is called an ( $i$-)information set.

- $u_{i}: Z \rightarrow \mathrm{R}$ is a payoff function for player $i$, assigning a real number ( $i$ 's payoff) to each run of the game.

An augmented game is defined much like an extensive game. The only essential difference is that at each nonterminal history we not only determine the player moving but also her awareness level. There are also extra moves of nature that intuitively capture players' uncertainty regarding the awareness level of their opponents. Formally, given an extensive game $\Gamma=\left(N, M, H, P, f_{c},\left\{\mathcal{I}_{i}\right.\right.$ : $\left.i \in N\},\left\{u_{i}: i \in N\right\}\right)$, an augmented game based on $\Gamma$ is a tuple $\Gamma^{+}=\left(N^{+}, M^{+}, H^{+}, P^{+}, f_{c}^{+},\left\{\mathcal{I}_{i}^{+}\right.\right.$: $\left.i \in N^{+}\right\},\left\{u_{i}^{+}: i \in N^{+}\right\},\left\{A_{i}^{+}: i \in N^{+}\right\}$), where $\left(N^{+}, M^{+}, H^{+}, P^{+}, f_{c}^{+},\left\{\mathcal{I}_{i}^{+}: i \in N^{+}\right\},\left\{u_{i}^{+}: i \in\right.\right.$ $\left.N^{+}\right\}$) is a standard extensive game with perfect recall ${ }^{1}$ and $A_{i}^{+}: H_{i}^{+} \rightarrow 2^{H}$ describes $i$ 's awareness level at each history at which he moves. $\Gamma^{+}$must satisfy some consistency conditions. These conditions basically ensure that a player's awareness level depends only on the information she has; players do not forget histories that they were aware of; and there is common knowledge of (1) what the payoffs are in the underlying game and (2) what the information sets are in the underlying game. The formal conditions are not needed in this paper, so we omit them here.

An augmented game describes either the modeler's view of the game or the subjective view of the game of one of the players, and includes both moves of the underlying game and moves of nature that change awareness. A game with awareness collects all these different views, and describes, in each view, what view other players have. Formally, a game with awareness based on $\Gamma=\left(N, M, H, P, f_{c},\left\{\mathcal{I}_{i}\right.\right.$ : $\left.i \in N\},\left\{u_{i}: i \in N\right\}\right)$ is a tuple $\Gamma^{*}=\left(\mathcal{G}, \Gamma^{m}, \mathcal{F}\right)$, where

\footnotetext{
${ }^{1} \mathrm{~A}$ game with perfect recall is one where, players remember all the actions they have performed and all the information sets they have passed through; see [Osborne and Rubinstein 1994] for the formal definition.
}

- $\mathcal{G}$ is a countable set of augmented games based on $\Gamma$, of which one is $\Gamma^{m}$;

- $\mathcal{F}$ maps an augmented game $\Gamma^{+} \in \mathcal{G}$ and a history $h$ in $\Gamma^{+}$such that $P^{+}(h)=i$ to a pair $\left(\Gamma^{h}, I\right)$, where $\Gamma^{h} \in \mathcal{G}$ and $I$ is an $i$-information set in game $\Gamma^{h}$.

Intuitively, $\Gamma^{m}$ is the game from the point of view of an omniscient modeler. If player $i$ moves at $h$ in game $\Gamma^{+} \in \mathcal{G}$ and $\mathcal{F}\left(\Gamma^{+}, h\right)=\left(\Gamma^{h}, I\right)$, then $\Gamma^{h}$ is the game that $i$ believes to be the true game when the history is $h$, and $I$ consists of the set of histories in $\Gamma^{h}$ that $i$ currently considers possible.

The augmented game $\Gamma^{m}$ and the mapping $\mathcal{F}$ must satisfy a number of consistency conditions. The conditions on the modeler's game ensures that the modeler is aware of all the players and moves of the underlying game, and that he understands how nature's moves work in the underlying game $\Gamma$. The game $\Gamma^{m}$ can be thought of as a description of "reality"; it describes the effect of moves in the underlying game and how players' awareness levels change. The other games in $\mathcal{G}$ describe a player's subjective view of the situation.

There are also ten constraints on the mapping $\mathcal{F}$ that capture desirable properties of awareness. Rather than describing all ten constraints here, we briefly describe a few of them, to give some idea of the intuition behind these constraints. Suppose that $\mathcal{F}\left(\Gamma^{+}, h\right)=\left(\Gamma^{h}, I\right)$ and $A_{i}^{+}(h)=$ $a,{ }^{2}$ then the following conditions hold.

C1. $\left\{\bar{h}: h \in H^{h}\right\}=a$, where $\bar{h}$ is the subsequence of $h$ consisting of all the moves in $h$ that are also in the set of moves $M$ of the underlying game $\Gamma$.

C2. If $h^{\prime} \in H^{h}$ and $P^{h}\left(h^{\prime}\right)=j$, then $A_{j}^{h}\left(h^{\prime}\right) \subseteq a$ and $M_{\bar{h}^{\prime}} \cap\left\{m: \bar{h}^{\prime} \cdot\langle m\rangle \in a\right\}=M_{h^{\prime}}^{h}$.

C5. If $h^{\prime} \in H^{+}, P^{+}\left(h^{\prime}\right)=i, A_{i}^{+}\left(h^{\prime}\right)=a$, then if $h$ and $h^{\prime}$ are in the same information set of $\Gamma^{+}$, then $\mathcal{F}\left(\Gamma^{+}, h^{\prime}\right)=\left(\Gamma^{h}, I\right)$, while if $h$ is a prefix or a suffix of $h^{\prime}$, then $\mathcal{F}\left(\Gamma^{+}, h^{\prime}\right)=\left(\Gamma^{h}, I^{\prime}\right)$ for some $i$ information set $I^{\prime}$.

C8. For all histories $h^{\prime} \in I$, there exists a prefix $h_{1}^{\prime}$ of $h^{\prime}$ such that $P^{h}\left(h_{1}^{\prime}\right)=i$ and $\mathcal{F}\left(\Gamma^{h}, h_{1}^{\prime}\right)=\left(\Gamma^{\prime}, I^{\prime}\right)$ iff there exists a prefix $h_{1}$ of $h$ such that $P^{+}\left(h_{1}\right)=i$ and $\mathcal{F}\left(\Gamma^{+}, h_{1}\right)=\left(\Gamma^{\prime}, I^{\prime}\right)$. Moreover, $h_{1}^{\prime} \cdot\langle m\rangle$ is a prefix of $h^{\prime}$ iff $h_{1} \cdot\langle m\rangle$ is a prefix of $h$.

C9. There exists a history $h^{\prime} \in I$ such that for every prefix $h^{\prime \prime} \cdot\langle m\rangle$ of $h^{\prime}$, if $P^{h}\left(h^{\prime \prime}\right)=j \in N^{h}$ and $\mathcal{F}\left(\Gamma^{h}, h^{\prime \prime}\right)=$ $\left(\Gamma^{\prime}, I^{\prime}\right)$, then for all $h_{1} \in I^{\prime}, h_{1} \cdot\langle m\rangle \in H^{\prime}$.

\footnotetext{
${ }^{2}$ As in our earlier paper, we use the convention that the components of a (standard or augmented) game $\Gamma^{+}$are labeled with the same superscript + , so that we have $M^{+}, H^{+}, A_{i}^{+}$, and so on.
} 
Suppose that $\mathcal{F}\left(\Gamma^{+}, h\right)=\left(\Gamma^{h}, I\right)$. Player $i$ moving at history $h$ in $\Gamma^{+}$thinks the actual game is $\Gamma^{h}$. Moreover, $i$ thinks he is in the information set of $I$ of $\Gamma^{h}$. C1 guarantees that the set of histories of the underlying game player $i$ is aware of is exactly the set of histories of the underlying game that appear in $\Gamma^{h}$. C2 states that no player in $\Gamma^{h}$ can be aware of histories not in $a$. The second part of C2 implies that the set of moves available for player $j$ at $h^{\prime}$ is just the set of moves that player $i$ is aware of that are available for $j$ at $\bar{h}^{\prime}$ in the underlying game. C5 says that player $i$ 's subjective view of the game changes only if $i$ becomes aware of more moves and is the same at histories in $\mathrm{H}^{+}$ that $i$ cannot distinguish.

C8 is a consequence of the perfect recall assumption. C8 says that if, at history $h, i$ considers $h^{\prime}$ possible, then for every prefix $h_{1}^{\prime}$ of $h^{\prime}$ there is a corresponding prefix of $h$ where $i$ considers himself to be playing the same game, and similarly, for every prefix of $h$ there is a prefix of $h^{\prime}$ where $i$ considers himself to be playing the same game. Moreover, $i$ makes the same move at these prefixes. The intuition behind condition $\mathrm{C} 9$ is that player $i$ knows that player $j$ only make moves that $j$ is aware of. Therefore, player $i$ must consider at least one history $h^{\prime}$ where he believes that every player $j$ made a move that $j$ was aware of. It follows from conditions on augmented games, C1, C2, and C9 that there is a run going through $I$ where every player $j$ makes a move that player $i$ believes that $j$ is aware of.

It may seem that by making $\mathcal{F}$ a function we cannot capture a player's uncertainty about the game being played or uncertainty about opponents' unawareness about histories. However, we can capture such uncertainty by folding it into nature's initial move in the game the player consider possible while moving. It should be clear that this gives a general approach to capturing such uncertainties.

We identify a standard extensive game $\Gamma$ with the game $\left(\left\{\Gamma^{m}\right\}, \Gamma^{m}, \mathcal{F}\right)$, where (abusing notation slightly) $\Gamma^{m}=$ $\left(\Gamma,\left\{A_{i}: i \in N\right\}\right)$ and, for all histories $h$ in an $i$ information set $I$ in $\Gamma, A_{i}(h)=H$ and $\mathcal{F}\left(\Gamma^{m}, h\right)=$ $\left(\Gamma^{m}, I\right)$. Thus, all players are aware of all the runs in $\Gamma$, and agree with each other and the modeler that the game is $\Gamma$. This is the canonical representation of $\Gamma$ as a game with awareness.

In [Halpern and Rêgo 2006], we discussed generalizations of games with awareness to include situations where players may be aware of their own unawareness and, more generally, games where players may not have common knowledge of the underlying game is; for example, players may disagree about what the payoffs or the information sets are. With these models, we can capture a situation where, for example, player $i$ may think that another player $j$ cannot make a certain a certain move, when in fact $j$ can make such a move. For ease of exposition, we do not discuss these generalizations further here. However, it is not hard to show that the results of this paper can be extended to them in a straightforward way.

Feinberg [2004, 2005] also studied games with awareness. Feinberg [2005] gives a definition of extended Nash equilibrium in normal-form games. Feinberg [2004] deals with extensive-form games and defines solution concepts only indirectly, via a syntactic epistemic characterization. His approach lacks a more direct semantic framework, which our model provides. Li [2006a] has also provided a model of unawareness in extensive games, based on her earlier work on modeling unawareness [Li 2006b; Li 2006c]. See [Halpern and Rêgo 2006] for some further discussion of the relation between these approaches and ours.

\section{GENERALIZED SEQUENTIAL EQUILIBRIUM}

To explain generalized sequential equilibrium, we first review the notion of sequential equilibrium for standard games. Sequential equilibrium is defined with respect to an assessment, a pair $(\vec{\sigma}, \mu)$ where $\vec{\sigma}$ is a strategy profile consisting of behavioral strategies and $\mu$ is a belief system, i.e., a function that determines for every information set $I$ a probability $\mu_{I}$ over the histories in $I$. Intuitively, if $I$ is an information set for player $i, \mu_{I}$ is $i$ 's subjective assessment of the relative likelihood of the histories in $I$. Roughly speaking, an assessment is a sequential equilibrium if for all players $i$, at every $i$-information set, (a) $i$ chooses a best response given the beliefs he has about the histories in that information set and the strategies of other players, and (b) $i$ 's beliefs are consistent with the strategy profile being played, in the sense that they are calculated by conditioning the probability distribution induced by the strategy profile over the histories on the information set.

Note that $\mu_{I}$ is defined even if $I$ is reached with probability 0 . Defining consistency at an information set that is reached with probability 0 is somewhat subtle. In that case, intuitively, once information set $I$ is reached player $i$ moving at $I$ must believe the game has been played according to an alternative strategy profile. In a sequential equilibrium, that alternative strategy profile consists of a small perturbation of the original assessment where every move is chosen with positive probability.

Given a strategy profile $\vec{\sigma}$, let $\operatorname{Pr}_{\vec{\sigma}}$ be the probability distribution induced by $\vec{\sigma}$ over the possible histories of the game. Intuitively, $\operatorname{Pr}_{\vec{\sigma}}(h)$ is the product of the probability of each of the moves in $h$. For simplicity we assume $f_{c}>0$, so that if $\vec{\sigma}$ is such that every player chooses all of his moves with positive probability, then for every history $h, \operatorname{Pr}_{\vec{\sigma}}(h)>0 .^{3}$ For any history $h$ of the game, define $\operatorname{Pr}_{\vec{\sigma}}(\cdot \mid h)$ to be the conditional probability distribution induced by $\vec{\sigma}$ over the

\footnotetext{
${ }^{3}$ See [Myerson 1991] for a definition of sequential equilibrium in the case nature chooses some of its move with probability 0 .
} 
possible histories of the game given that the current history is $h$. Intuitively, $\operatorname{Pr}_{\vec{\sigma}}\left(h^{\prime} \mid h\right)$ is 0 if $h$ is not a prefix of $h^{\prime}$, is 1 if $h=h^{\prime}$, and is the product of the probability of each of the moves in the path from $h$ to $h^{\prime}$ if $h$ is a prefix of $h^{\prime}$. Formally, an assessment $(\vec{\sigma}, \mu)$ is a sequential equilibrium if it satisfies the following properties:

- Sequential rationality. For every information set $I$ and player $i$ and every behavioral strategy $\sigma$ for player $i$,

$$
\mathrm{EU}_{i}((\vec{\sigma}, \mu) \mid I) \geq \mathrm{EU}_{i}\left(\left(\left(\vec{\sigma}_{-i}, \sigma\right), \mu\right) \mid I\right),
$$

where $\operatorname{EU}_{i}((\vec{\sigma}, \mu) \mid I)=\sum_{h \in I} \sum_{z \in Z} \mu_{I}(h) \operatorname{Pr}_{\vec{\sigma}}(z \mid$ h) $u_{i}(z)$.

- Consistency between belief system and strategy profile. If $\vec{\sigma}$ consists of completely mixed (behavior) strategies, that is, ones that assign positive probability to every action at every information set, then for every information set $I$ and history $h$ in $I$,

$$
\mu_{I}(h)=\frac{\operatorname{Pr}_{\vec{\sigma}}(h)}{\sum_{h^{\prime} \in I} \operatorname{Pr}_{\vec{\sigma}}\left(h^{\prime}\right)} .
$$

Otherwise, there exists a sequence $\left(\vec{\sigma}^{n}, \mu^{n}\right), n=$ $1,2,3, \ldots$, of assessments such that $\vec{\sigma}^{n}$ consists of completely mixed strategies, $\left(\vec{\sigma}^{n}, \mu^{n}\right)$ is consistent in the above sense, and $\lim _{n \rightarrow \infty}\left(\vec{\sigma}^{n}, \mu^{n}\right)=(\vec{\sigma}, \mu)$.

In order to define a generalized notion of sequential equilibrium for games with awareness, we first need to define a generalized notion of assessment for games with awareness. We first need a generalized notion of strategy, which we defined in our earlier paper.

Intuitively, a strategy describes what $i$ will do in every possible situation that can arise. This no longer makes sense in games with awareness, since a player no longer understands in advance all the possible situations that can arise. For example, player $i$ cannot plan in advance for what will happen if he becomes aware of something he is initially unaware of. We solved this problem in our earlier paper as follows. Let $\mathcal{G}_{i}=\left\{\Gamma^{\prime} \in \mathcal{G}\right.$ : for some $\Gamma^{+} \in$ $\mathcal{G}$ and $h$ in $\Gamma^{+}, P^{+}(h)=i$ and $\left.\mathcal{F}\left(\Gamma^{+}, h\right)=\left(\Gamma^{\prime}, \cdot\right)\right\}$. Intuitively, $\mathcal{G}_{i}$ consists of the games that $i$ views as the real game in some history. Rather than considering a single strategy in a game $\Gamma^{*}=\left(\mathcal{G}, \Gamma^{m}, \mathcal{F}\right)$ with awareness, we considered a collection $\left\{\sigma_{i, \Gamma^{\prime}}: \Gamma^{\prime} \in \mathcal{G}_{i}\right\}$ of local strategies. Intuitively, a local strategy $\sigma_{i, \Gamma^{\prime}}$ for game $\Gamma^{\prime}$ is the strategy that $i$ would use if $i$ were called upon to play and $i$ thought that the true game was $\Gamma^{\prime}$. Thus, the domain of $\sigma_{i, \Gamma^{\prime}}$ consists of pairs $\left(\Gamma^{+}, h\right)$ such that $\Gamma^{+} \in \mathcal{G}, h$ is a history in $\Gamma^{+}, P^{+}(h)=i$, and $\mathcal{F}\left(\Gamma^{+}, h\right)=\left(\Gamma^{\prime}, I\right)$. Let $\left(\Gamma^{h}, I\right)^{*}=\left\{\left(\Gamma^{\prime}, h\right): \mathcal{F}\left(\Gamma^{\prime}, h\right)=\left(\Gamma^{h}, I\right)\right\}$; we call $\left(\Gamma^{h}, I\right)^{*}$ a generalized information set.

Definition 3.1: Given a game $\Gamma^{*}=\left(\mathcal{G}, \Gamma^{m}, \mathcal{F}\right)$ with awareness, a local strategy $\sigma_{i, \Gamma^{\prime}}$ for agent $i$ is a function mapping pairs $\left(\Gamma^{+}, h\right)$ such that $h$ is a history where $i$ moves in $\Gamma^{+}$and $\mathcal{F}\left(\Gamma^{+}, h\right)=\left(\Gamma^{\prime}, I\right)$ to a probability distribution over $M_{h^{\prime}}^{\prime}$, the moves available at a history $h^{\prime} \in I$, such that $\sigma_{i, \Gamma^{\prime}}\left(\Gamma_{1}, h_{1}\right)=\sigma_{i, \Gamma^{\prime}}\left(\Gamma_{2}, h_{2}\right)$ if $\left(\Gamma_{1}, h_{1}\right)$ and $\left(\Gamma_{2}, h_{2}\right)$ are in the same generalized information set. A generalized strategy profile of $\Gamma^{*}=\left(\mathcal{G}, \Gamma^{m}, \mathcal{F}\right)$ is a set of local strategies $\vec{\sigma}=\left\{\sigma_{i, \Gamma^{\prime}}: i \in N, \Gamma^{\prime} \in \mathcal{G}_{i}\right\}$. A generalized belief system $\mu$ is a function that associates each generalized information set $\left(\Gamma^{\prime}, I\right)^{*}$ with a probability distribution $\mu_{\Gamma^{\prime}, I}$ over the set $\left\{\left(\Gamma^{\prime}, h\right): h \in I\right\}$. A generalized assessment is a pair $(\vec{\sigma}, \mu)$, where $\vec{\sigma}$ is a generalized strategy profile and $\mu$ is a generalized belief system.

We can now define what it means for a generalized assessment $\left(\vec{\sigma}^{*}, \mu^{*}\right)$ to be a generalized sequential equilibrium of a game with awareness. The definition is essentially identical to that of $(\vec{\sigma}, \mu)$ being a sequential equilibrium; the use of $\mathrm{EU}_{i}$ in the definition of sequential rationality is replaced by $\mathrm{EU}_{i, \Gamma^{\prime}}$, where $\mathrm{EU}_{i, \Gamma^{\prime}}\left(\left(\vec{\sigma}^{*}, \mu^{*}\right) \mid I\right)$ is the conditional expected payoff for $i$ in the game $\Gamma^{\prime}$, given that strategy profile $\vec{\sigma}^{*}$ is used, information set $I$ has been reached, and player $i$ 's beliefs about the histories in $I$ are described by $\mu_{\Gamma^{\prime}, I}^{*}$. We leave the straightforward modifications of the definition to the reader. It is easy to see that $(\vec{\sigma}, \mu)$ is a sequential equilibrium of a standard game $\Gamma$ iff $(\vec{\sigma}, \mu)$ is a (generalized) sequential equilibrium of the canonical representation of $\Gamma$ as a game with awareness. Thus, our definition of generalized sequential equilibrium generalizes the standard definition.

To better understand the concept of generalized sequential equilibrium concept, consider the game shown in Figure 2. Suppose that both players 1 and 2 are aware of all runs of the game, but player 1 (falsely) believes that player 2 is aware only of the runs not involving $L$ and believes that player 1 is aware of these runs as well. Also suppose that player 2 is aware of all of this; that is, player 2's view of the game is the same as the modeler's view of the game $\Gamma^{m}$ shown in Figure 2. While moving at node 1.1, player 1 considers the true game to be identical to the modeler's game except that from player 1's point of view, while moving at 2.1, player 2 believes the true game is $\Gamma^{2.2}$, shown in Figure 3.

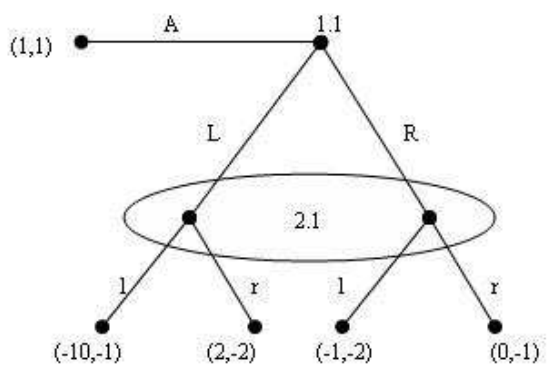

Figure 2: The modeler's game $\Gamma^{m}$. 
$(1,1)$

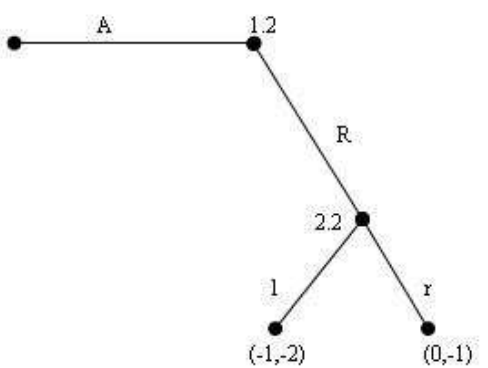

Figure 3: Player 2's view of the game from point of view of player 1 .

This game has a unique generalized sequential equilibrium where player 2 chooses $r$ and player 1 chooses $A$ in $\Gamma^{2.2}$. Believing that player 2 will move $r$, player 1 best responds by choosing $L$ at node 1.1. Since player 2 knows all this at node 2.1 in $\Gamma^{m}$, she chooses $l$ at this node. Thus, if players follow their equilibrium strategies, the payoff vector is $(-10,-1)$. In this situation, player 2 would be better off is she could let player 1 know that she is aware of move $L$, since then player 1 would play $A$ and both players would receive 1 . On the other hand, if we slightly modify the game by making $u_{2}(\langle L, l\rangle)=3$, then player 2 would benefit from the fact that 1 believes that she is unaware of move $L$.

We now want to show that every game with awareness $\Gamma^{*}$ has at least one generalized sequential equilibrium. To prove that a game with awareness $\Gamma^{*}$ has a generalized Nash equilibrium, we constructed a standard game $\Gamma^{\nu}$ with perfect recall and showed that there exists a one-to-one correspondence between the set of generalized Nash equilibrium of $\Gamma^{*}$ and the set of Nash equilibrium of $\Gamma^{\nu}$. Intuitively, $\Gamma^{\nu}$ is constructed by essentially "gluing together" all the games $\Gamma^{\prime} \in \mathcal{G}$, except that only histories in $\Gamma^{\prime}$ that can actually be played according to the players' awareness level are considered.

More formally, given a game $\Gamma^{*}=\left(\mathcal{G}, \Gamma^{m}, \mathcal{F}\right)$ with awareness, let $\nu$ be a probability on $\mathcal{G}$ that assigns each game in $\mathcal{G}$ positive probability. (Here is where we use the fact that $\mathcal{G}$ is countable.) For each $\Gamma^{\prime} \in \mathcal{G}$, let $\left\lfloor H^{\Gamma^{\prime}}\right\rfloor=\left\{h \in H^{\Gamma^{\prime}}\right.$ : for every prefix $h_{1} \cdot\langle m\rangle$ of $h$, if $P^{\prime}\left(h_{1}\right)=i \in N$ and $\mathcal{F}\left(\Gamma^{\prime}, h_{1}\right)=\left(\Gamma^{\prime \prime}, I\right)$, then for all $\left.h_{2} \in I, h_{2} \cdot\langle m\rangle \in H^{\prime \prime}\right\}$. The histories in $\left\lfloor H^{\Gamma^{\prime}}\right\rfloor$ are the ones that can actually be played according to the players' awareness levels.

Let $\Gamma^{\nu}$ be the standard game such that

- $N^{\nu}=\left\{\left(i, \Gamma^{\prime}\right): \Gamma^{\prime} \in \mathcal{G}_{i}\right\}$

- $M^{\nu}=\mathcal{G} \cup \cup_{\Gamma^{\prime} \in \mathcal{G}}\left\lfloor M^{\Gamma^{\prime}}\right\rfloor$, where $\left\lfloor M^{\Gamma^{\prime}}\right\rfloor$ is the set of moves that occur in $\left\lfloor H^{\Gamma^{\prime}}\right\rfloor$;

- $H^{\nu}=\langle\rangle \cup\left\{\left\langle\Gamma^{\prime}\right\rangle \cdot h: \Gamma^{\prime} \in \mathcal{G}, h \in\left\lfloor H^{\Gamma^{\prime}}\right\rfloor\right\}$;
- $P^{\nu}(\langle\rangle)=c$, and

$$
P^{\nu}\left(\left\langle\Gamma^{h}\right\rangle \cdot h^{\prime}\right)= \begin{cases}\left(i, \Gamma^{h^{\prime}}\right) & \text { if } P^{h}\left(h^{\prime}\right)=i \in N \text { and } \\ & \mathcal{F}\left(\Gamma^{h}, h^{\prime}\right)=\left(\Gamma^{h^{\prime}}, \cdot\right), \\ c & \text { if } P^{h}\left(h^{\prime}\right)=c ;\end{cases}
$$

- $f_{c}^{\nu}\left(\Gamma^{\prime} \mid\langle\rangle\right)=\nu\left(\Gamma^{\prime}\right)$ and $f_{c}^{\nu}\left(\cdot \mid\left\langle\Gamma^{h}\right\rangle \cdot h^{\prime}\right)=f_{c}^{h}\left(\cdot \mid h^{\prime}\right)$ if $P^{h}\left(h^{\prime}\right)=c$

- $\mathcal{I}_{i, \Gamma^{\prime}}^{\nu}$ is a partition of $H_{i, \Gamma^{\prime}}^{\nu}$ where two histories $\left\langle\Gamma^{1}\right\rangle$. $h^{1}$ and $\left\langle\Gamma^{1}\right\rangle \cdot h^{1}$ are in the same information set $\left\langle\Gamma^{\prime}, I\right\rangle^{*}$ iff $\left(\Gamma^{1}, h^{1}\right)$ and $\left(\Gamma^{2}, h^{2}\right)$ are in the same generalized information set $\left(\Gamma^{\prime}, I\right)^{*}$;

- $u_{i, \Gamma^{\prime}}^{\nu}\left(\left\langle\Gamma^{h}\right\rangle \cdot z\right)= \begin{cases}u_{i}^{h}(z) & \text { if } \Gamma^{h}=\Gamma^{\prime} \\ 0 & \text { if } \Gamma^{h} \neq \Gamma^{\prime}\end{cases}$

Unfortunately, while it is the case that there is a 1-1 correspondence between the Nash equilibria of $\Gamma^{\nu}$ and the generalized Nash equilibria of $\Gamma^{*}$, as we show in the full paper, this correspondence breaks down for sequential equilibria. Thus, we need to take a different approach to showing that a generalized sequential equilibrium always exists.

\section{CONDITIONAL SEQUENTIAL EQUILIBRIUM}

In the standard definition of sequential equilibrium for extensive games, it is implicitly assumed that every player considers all histories in his information set possible. This is evident from the fact that if a strategy profile that is part of a sequential equilibrium assigns positive probability to every move, then by the consistency requirement the belief system also assigns positive probability to every history of every information set of the game. Therefore, this notion of equilibrium is not strong enough to capture situations where a player is certain that some histories in his information set will not occur. The notion of conditional sequential equilibrium, which we now define, is able to deal with such situations. It generalizes sequential equilibrium: in a game where every player considers every history in his information set possible, the set of conditional sequential equilibria and the set of sequential equilibria coincide.

Given a standard extensive game $\Gamma$, define a possibility system $\mathcal{K}$ on $\Gamma$ to be a function that determines for every information set $I$ a nonempty subset of $I$ consisting of the histories in $I$ that the player moving at $I$ considers possible. We assume that $\mathcal{K}$ is common knowledge among players of the game, so that every player understands what histories are considered possible by everyone else in the game. If $I$ is an $i$-information set, intuitively $i$ should be indifferent among all runs that go through histories in $I-\mathcal{K}(I)$, since $i$ believes that those runs will not occur and every other player knows that. Thus, for a given $\Gamma$, a possibility system $\mathcal{K}$ must satisfy the following requirement: if $z$ and $z^{\prime}$ are 
two runs going through histories in $I-\mathcal{K}(I)$ and $I$ is an $i$-information set, then $u_{i}(z)=u_{i}\left(z^{\prime}\right)$.

Given a pair $(\Gamma, \mathcal{K})$, a $\mathcal{K}$-assessment is a pair $(\vec{\sigma}, \mu)$, where $\vec{\sigma}$ is a strategy profile of $\Gamma$, and $\mu$ is a restricted belief system, i.e., a function that determines for every information set $I$ of $\Gamma$ a probability $\mu_{I}$ over the histories in $\mathcal{K}(I)$. Intuitively, if $I$ is an information set for player $i, \mu_{I}$ is $i$ 's subjective assessment of the relative likelihood of the histories player $i$ considers possible while moving at $I$, namely $\mathcal{K}(I)$. As in the definition of sequential equilibrium, a $\mathcal{K}$ assessment $(\vec{\sigma}, \mu)$ is a conditional sequential equilibrium with respect to $\mathcal{K}$ if (a) at every information set where a player moves he chooses a best response given the beliefs he has about the histories that he considers possible in that information set and the strategies of other players, and (b) his restricted beliefs must be consistent with the strategy profile being played and the possibility system, in the sense that they are calculated by conditioning the probability distribution induced by the strategy profile over the histories considered possible on the information set. Formally, the definition of $(\vec{\sigma}, \mu)$ being a conditional sequential equilibrium is identical to that of sequential equilibrium, except that the summation in the definition of $\left.\mathrm{EU}_{i}(\vec{\sigma}, \mu) \mid I\right)$ and $\mu_{I}(h)$ is taken over histories in $\mathcal{K}(I)$ rather than histories in $I$. It is immediate that if $\mathcal{K}(I)=I$ for every information set $I$ of the game, then the set of conditional sequential equilibria with respect to $\mathcal{K}$ coincides with the set of sequential equilibria. The next theorem shows that set of conditional sequential equilibria for a large class of extensive games that includes $\Gamma^{\nu}$ is nonempty.

Theorem 4.1: Let $\Gamma$ be an extensive game with perfect recall and countably many players such that (a) each player has only finitely many pure strategies and (b) each player's payoff depends only on the strategy of finitely many other players. Let $\mathcal{K}$ be an arbitrary possibility system. Then there exists at least one $\mathcal{K}$-assessment that is a conditional sequential equilibrium of $\Gamma$ with respect to $\mathcal{K}$.

We now prove that every game of awareness has a generalized sequential equilibrium by defining a possibility system $\mathcal{K}$ on $\Gamma^{\nu}$ and showing that there is a one-to-one correspondence between the set of conditional sequential equilibria of $\Gamma^{\nu}$ with respect to $\mathcal{K}$ and the set of generalized sequential equilibria of $\Gamma^{*}$.

Theorem 4.2 : For all probability measures $\nu$ on $\mathcal{G}$, if $\nu$ gives positive probability to all games in $\mathcal{G}$, and $\mathcal{K}\left(\left\langle\Gamma^{\prime}, I\right\rangle^{*}\right)=\left\{\left\langle\Gamma^{\prime}, h\right\rangle: h \in I\right\}$ for every information set $\left\langle\Gamma^{\prime}, I\right\rangle^{*}$ of $\Gamma^{\nu}$, then $\left(\vec{\sigma}^{\prime}, \mu^{\prime}\right)$ is a generalized sequential equilibrium of $\Gamma^{*}$ iff $(\vec{\sigma}, \mu)$ is a conditional sequential equilibrium of $\Gamma^{\nu}$ with respect to $\mathcal{K}$, where $\sigma_{i, \Gamma^{\prime}}\left(\left\langle\Gamma^{h}\right\rangle \cdot h^{\prime}\right)=$ $\sigma_{i, \Gamma^{\prime}}^{\prime}\left(\Gamma^{h}, h^{\prime}\right)$ and $\mu_{\Gamma^{\prime}, I}^{\prime}=\mu_{\left\langle\Gamma^{\prime}, I\right\rangle^{*}}$.

Since $\Gamma^{\nu}$ satisfies all the conditions of Theorem 4.1, it easily follows from Theorems 4.1 and 4.2 that every game with awareness has at least one generalized sequential equilibrium.

Although it is not true that every conditional sequential equilibrium is also a sequential equilibrium of an arbitrary game, the next theorem shows there is a close connection between these notions of equilibrium. If $(\vec{\sigma}, \mu)$ is a conditional sequential equilibrium with respect to some possibility system $\mathcal{K}$, then there exists a belief system $\mu^{\prime}$ such that $\left(\vec{\sigma}, \mu^{\prime}\right)$ is a sequential equilibrium.

Theorem 4.3: For every extensive game $\Gamma$ with countably many players where each player has finitely many pure strategies and for every possibility system $\mathcal{K}$, if $(\vec{\sigma}, \mu)$ is a conditional sequential equilibrium of $\Gamma$ with respect to $\mathcal{K}$, then there exists a belief system $\mu^{\prime}$ such that $\left(\vec{\sigma}, \mu^{\prime}\right)$ is a sequential equilibrium of $\Gamma$.

\section{RATIONALIZABILITY AND GENERALIZED NASH EQUILIBRIUM}

In this section, we analyze the relationship between the notions of rationalizability and generalized Nash equilibrium, providing some more intuition about the latter.

The usual justification for Nash equilibrium is that a player's strategy must be a best response to the strategies selected by other players in the equilibrium, because he can deduce what those strategies are. However, in most strategic situations, it is not the case that a player can deduce the strategies used by other players. Since every player tries to maximize his expected payoff and this is common knowledge, the best that a player can hope to do is to deduce a set of reasonable strategies for the other players. Here, we take a "reasonable strategy" to be a best response to some reasonable beliefs a player might hold about the strategy profile being played. This is the intuition that the rationalizability solution concept tries to capture. Even though a notion of rationalizability for extensive-form games was proposed by Pearce [1984], rationalizability is more widely applied in normal-form games. In this section, we explore the relationship between rationalizability and generalized Nash equilibrium in games with awareness where in the underlying game each player moves only once, and these moves are made simultaneously (or, equivalently, a player does not know the moves made by other players before making his own move). We show that, given an underlying game $\Gamma$ satisfying this requirement, a pure strategy profile contains only rationalizable strategies iff it is the strategy profile used by the players in the modeler's game in some (pure) generalized Nash equilibrium of a game $\Gamma^{*}$ with awareness. If we think of rationalizability as characterizing "best response to your beliefs" and Nash equilibrium characterizing "best response to what is actually played", then this result shows that in the framework of games with awareness, since the game is not common knowledge, the 
line between these two notions is somewhat blurred.

We start by reviewing the notion of rationalizability for standard normal-form games. Let $\mathcal{C}_{i}$ be the set of available pure strategies for player $i$; $\mathcal{C}=\times_{i \in N} \mathcal{C}_{i}$ is thus the set of pure strategy profiles. Let $\Delta(M)$ denote the set of all probability distributions on $M$. Suppose that each player $i$ is rational and is commonly known to choose a strategy from a subset $\mathcal{D}_{i}$ of $\mathcal{C}_{i}$. Let $\mathcal{D}_{-i}=\times_{j \neq i} \mathcal{D}_{i}$ and

$$
\begin{aligned}
& B\left(\mathcal{D}_{-i}\right)=\left\{\operatorname{argmax}_{s_{i} \in \mathcal{C}_{i}} \mathrm{EU}_{i}\left(\left(s_{i}, \pi\left(\mathcal{D}_{-i}\right)\right)\right):\right. \\
& \text { for some } \left.\pi \in \Delta\left(\mathcal{D}_{-i}\right)\right\} ;
\end{aligned}
$$

that is, $B\left(\mathcal{D}_{-i}\right)$ consists of the strategies in $\mathcal{C}_{i}$ that are best responses to some belief that player $i$ could have about the strategies other players are using.

The set $\mathcal{S}=\times_{i \in N} \mathcal{S}_{i}$ of correlated rationalizable strategies is characterized by the following two properties: (a) for all $i \in N, \mathcal{S}_{i} \subseteq B\left(\mathcal{S}_{-i}\right)$ and (b) $\mathcal{S}$ is the largest set satisfying condition (a), in the sense that, for every set of strategy profiles $\mathcal{D}$ satisfying (a), we have that $\mathcal{D} \subseteq \mathcal{S}$. It is not hard to show that for every player $i, \mathcal{S}_{i}=B\left(\mathcal{S}_{-i}\right)$. A strategy $s_{i} \in \mathcal{S}_{i}$ is called a correlated rationalizable strategy for player $i^{4}{ }^{5}$

It turns out that we can construct $\mathcal{S}$ by the following iterative procedure. Let $\mathcal{C}_{i}^{0}=\mathcal{C}_{i}$ for all $i \in N$. Define $\mathcal{C}_{i}^{j}=B\left(\mathcal{C}_{-i}^{j-1}\right)$ for $j \geq 1$. Since there are finitely many strategies it is easy to see that there exists a finite $k$ such that $\mathcal{C}_{i}^{j}=\mathcal{C}_{i}^{k}$ for all $j \geq k$. It can be shown that $\mathcal{S}_{i}=\lim _{j \rightarrow \infty} \mathcal{C}_{i}^{j}=\mathcal{C}_{i}^{k}$. It is also easy to see that if $\vec{\sigma}$ is a (behavioral) Nash equilibrium, then every pure strategy that is played with positive probability according to $\vec{\sigma}$ is rationalizable (where the probability with which a pure strategy is played according to $\vec{\sigma}$ is the product of the probability of each of its moves according to $\vec{\sigma}$ ).

We now explore the relationship between rationalizability in an underlying game $\Gamma$ in normal-form and generalized Nash equilibrium in a special class of games with awareness based on $\Gamma$. Given a standard game $\Gamma$ and a pure strategy profile $\vec{s}$ consisting of rationalizable strategies, we define a game with awareness $\Gamma^{*}(\vec{s})=\left(\mathcal{G}, \Gamma^{m}, \mathcal{F}\right)$ such that (a) there exists a generalized Nash equilibrium of $\Gamma^{*}(\vec{s})$, where $s_{i}$ is the strategy followed by player $i$ in $\Gamma^{m}$, and (b) every local strategy in every pure generalized Nash equilibrium of $\Gamma^{*}(\vec{s})$ is rationalizable in $\Gamma$. To understand the intuition behind the construction, note that if $s_{i}$ is a pure

\footnotetext{
${ }^{4}$ From now on, we use $\vec{s}=\left(s_{1}, \ldots, s_{n}\right)$ to denote pure strategy profiles, and will continue to use $\vec{\sigma}$ for possibly nonpure strategy profiles.

${ }^{5}$ In the literature, it is often assumed that each player chooses his strategy independently of the others and that this is common knowledge. If we make this assumption, we get a somewhat stronger solution concept (at least, if $|N| \geq 3$ ), which we call uncorrelated rationalizability. Essentially the same results as we prove here for correlated rationalizability hold for uncorrelated rationalizability; we omit further details here.
}

correlated rationalizable strategy of player $i$ in $\Gamma$, then $s_{i}$ must be a best response to some probability distribution $\pi^{s_{i}}$ over the set $\mathcal{S}_{-i}$ of pure correlated rationalizable strategies of $i$ 's opponents. The idea will be to include a game $\Gamma^{s_{i}}$ in $\mathcal{G}$ that captures the beliefs that make $s_{i}$ a best response. Let $\vec{s}^{1}, \ldots, \vec{s}^{m}$ be the strategy profiles in $\mathcal{S}_{-i}$ that get positive probability according to $\pi^{s_{i}}$. (There are only finitely many, since $\mathcal{S}_{-i}$ consists of only pure strategies.) Let $\Gamma^{s_{i}}$ be the game where nature initially makes one of $m$ moves, say $c_{1}, \ldots, c_{m}$ (one corresponding to each strategy that gets positive probability according to $\pi^{s_{i}}$ ), where the probability of move $c_{j}$ is $\pi^{s_{i}}\left(\vec{s}^{j}\right)$. After nature's choice a copy of $\Gamma$ is played. All the histories in $\Gamma^{s_{i}}$ in which player $i$ is about to move are in the same information set of player $i$; that is, player $i$ does not know nature's move. However, all the other players know nature's move. Finally, all players are aware of all runs of $\Gamma$ at every history in $\Gamma^{s_{i}}$. Note that if $h$ is a history where player $i$ thinks game $\Gamma^{s_{i}}$ is the actual game, and believes that other players will play $\vec{s}_{-i}^{j}$ after nature's move $c_{j}$, then player $i$ believes that $s_{i}$ is a best response at $h$.

Given a pure strategy profile $\vec{s}$ of the game $\Gamma$, let $\Gamma^{*}(\vec{s})=$ $\left(\mathcal{G}, \Gamma^{m}, \mathcal{F}\right)$ be the following game with awareness:

- $\Gamma^{m}=\left(\Gamma,\left\{A_{i}: i \in N\right\}\right)$, where for every player $i$ and every history $h \in H_{i}^{m}, A_{i}(h)=H$ (the set of all histories in $\Gamma$ );

- $\mathcal{G}=\left\{\Gamma^{m}\right\} \cup\left\{\Gamma^{s_{i}^{\prime}}: s_{i}^{\prime} \in \mathcal{S}_{i}, i \in N\right\}$;

- for an augmented game in $\Gamma^{+} \in\left(\mathcal{G}-\left\{\Gamma^{m}\right\}\right)$ and a history $h$ of $\Gamma^{+}$of the form $\left\langle s_{-i}^{\prime}\right\rangle \cdot h^{\prime}$,

- if $P^{+}(h)=i$, then $\mathcal{F}\left(\Gamma^{+}, h\right)=\left(\Gamma^{+}, I\right)$ where $I$ is the information set containing $h$;

- if $P^{+}(h)=j \in N-\{i\}$ and $s_{j}^{\prime}$ is the strategy of player $j$ specified by $s_{-i}^{\prime}$, then $\mathcal{F}\left(\Gamma^{+}, h\right)=$ $\left(\Gamma^{s_{j}^{\prime}}, I\right)$, where $I$ is the unique $j$-information set in game $\Gamma^{s_{j}^{\prime}}$;

- for $h \in H_{i}^{m}, \mathcal{F}\left(\Gamma^{m}, h\right)=\left(\Gamma^{s_{i}}, I\right)$, where $I$ is the unique $i$-information set in game $\Gamma^{s_{i}}$.

The intuition is that if $\vec{s}$ is a strategy profile such that, for all $i \in N, s_{i}$ is a rationalizable strategy for player $i$ in $\Gamma$, then at the (unique) $i$-information set of $\Gamma^{m}, i$ considers the actual game to be $\Gamma^{s_{i}}$. For this particular game with awareness, there exists a generalized Nash equilibrium where the strategy for each player $i$ in the modeler's game is $s_{i}$. Conversely, only rationalizable strategies are used in any pure generalized Nash equilibrium of $\Gamma^{*}(\vec{s})$. There is only one small problem with this intuition: strategies in $\Gamma$ and local strategies for augmented games in $\Gamma^{*}(\vec{s})$ are defined over different objects. The former are defined over information sets of the underlying game $\Gamma$ and the latter are defined over generalized information sets of $\Gamma^{*}(s)$. Fortunately, 
this problem is easy to deal with: we can identify a local strategy in $\Gamma^{*}(\vec{s})$ with a strategy in $\Gamma$ in the obvious way. By definition of $\Gamma^{*}(\vec{s})$, for every player $i$ and augmented game $\Gamma^{\prime} \in \mathcal{G}_{i}$, the domain of the local strategy $\sigma_{i, \Gamma^{\prime}}$ consists of a unique generalized information set. We denote this information set by $I_{i, \Gamma^{\prime}}$. For each local strategy $\sigma_{i, \Gamma^{\prime}}$ of $\Gamma^{*}(\vec{s})$, we associate the strategy $\underline{\sigma}_{i, \Gamma^{\prime}}$ in the underlying game $\Gamma$ such that $\sigma_{i, \Gamma^{\prime}}\left(I_{i, \Gamma^{\prime}}\right)=\underline{\sigma}_{i, \Gamma^{\prime}}(I)$, where $I$ is the unique $i$-information set in $\Gamma$.

The following theorem summarizes the relationship between correlated rationalizable strategies in $\Gamma$ and generalized Nash equilibrium of games with awareness.

Theorem 5.1: If $\Gamma$ is a standard normal-form game and $\vec{s}$ is a (pure) strategy profile such that for all $i \in N, s_{i}$ is a correlated rationalizable strategy of player $i$ in $\Gamma$, then

(i) there is a (pure) generalized Nash equilibrium $\vec{s}^{*}$ of $\Gamma^{*}(\vec{s})$ such that for every player $i, \underline{s}_{i, \Gamma^{s_{i}}}^{*}=s_{i}$;

(ii) for every (pure) generalized Nash equilibrium $\vec{s}^{*}$ of $\Gamma^{*}(\vec{s})$, for every local strategy $s_{i, \Gamma^{\prime}}^{*}$ for every player $i$ in $\vec{s}^{*}$, the strategy $\underline{s}_{i, \Gamma^{\prime}}^{*}$ is correlated rationalizable for player $i$ in $\Gamma$.

Note that Theorem 5.1 does not imply that for a fixed game with awareness, (pure) generalized Nash equilibrium and generalized rationalizability coincide. These notions are incomparable for standard extensive games (cf. [Battigalli 1997; Pearce 1984]), so the corresponding generalized notions are incomparable when applied to the canonical representation of a standard game as a game with awareness. If we restrict the underlying game to be in normal form, it can be shown that, just as in standard games, every strategy in a pure generalized Nash equilibrium is (generalized) rationalizable. Since rationalizability is usually defined for pure strategies in the literature [Myerson 1991; Osborne and Rubinstein 1994], we focused on that case here. But it is not hard to show that an analogue of Theorem 5.1 holds for behavioral rationalizable strategies as well.

\section{DISCUSSION}

Our focus in this paper has been on refinements of generalized Nash equilibrium in games with awareness. It is worth reconsidering here the conceptual basis for these solution concepts in the presence of awareness. ${ }^{6}$ As we noted, equilibrium refinements are used in standard games to eliminate some "undesirable" or "unreasonable" equilibria. Arguably, unreasonable equilibria pose an even deeper problem with unaware players. For example, one standard interpretation of a Nash equilibrium is that a player chooses his

\footnotetext{
${ }^{6}$ We thank Aviad Heifetz and an anonymous referee for raising some of these issues.
}

strategy at the beginning of the game, and then does not change it because he has no motivation for doing so (since his payoff is no higher when he changes strategies). But this interpretation is suspect in extensive-form games when a player makes a move that that takes the game off the equilibrium path. It may seem unreasonable for a player to then play the move called for by his strategy (even if the strategy is part of a Nash equilibrium). A threat to blow up the world if I catch you cheating in a game may be part of a Nash equilibrium, and does not cause problems if in fact no one cheats, but it hardly seems credible if someone does cheat.

One way to justify the existence of incredible threats off the equilibrium path in a Nash equilibrium is to view the player as choosing a computer program that will play the game for him, and then leaving. Since the program is not changed once it is set in motion, threats about moves that will be made at information sets off the equilibrium path become more credible. However, in a game with awareness, a player cannot write a program to play the whole game at the beginning of the game because, when his level of awareness changes, he realizes that there are moves available to him that he was not aware of at the beginning of the game. He thus must write a new program that takes this into account. But this means we cannot sidestep the problem of incredible threats by appealing to the use of a pre-programmed computer to play a strategy. Once we allow a player to change his program, threats that were made credible because the program could not be rewritten become incredible again. Thus, the consideration of equilibrium refinements such as sequential equilibrium, which block incredible threats, becomes even more pertinent with awareness.

Moving up a level, we might ask more generally for the appropriate interpretation of Nash equilibrium in games with awareness. In standard games with a unique Nash equilibrium, we could perhaps argue that rational players will play their component of the equilibrium, since they can compute it and realize that it is the only stable strategy. In games with several Nash equilibria, perhaps one can be singled out as most salient, or some can be eliminated by using refinements of Nash equilibria.

To some extent, these considerations apply in games with awareness as well. If there is a unique generalized Nash equilibrium, although a player cannot necessarily compute the whole equilibrium (for example, if it involves moves that he is not aware of), he can compute that part of the equilibrium that is within the scope of his awareness. Thus, this argument for playing a Nash equilibrium lifts from standard games to games with awareness. However, other arguments do not lift so well. For example, in standard games, one argument for Nash equilibrium is that, over time, players will learn to play a Nash eqilibrium, for example, by playing a best response to their current beliefs. This 
argument will not work in the presence of awareness, since playing the game repeatedly can make players aware of moves or of other players awareness, and thus effectively change the game altogether.

Another way of interpreting Nash equilibrium in standard games is in terms of evolutionary game theory. This approach works with awareness as well. Suppose that we have populations consisting of each awareness type of each player, and that at each time step we draw without replacement one individual of each of these populations and let them play the game once. If the sample individuals are playing an equilibrium strategy, they do not have incentive to deviate unilaterally given their beliefs that the other players will continue to follow the equilibrium strategies.

Other issues arise when considering sequential equilibrium in games with awareness. For example, in a standard game, when a player reaches a history that is not on the equilibrium path, he must believe that his opponent made a mistake. However, in games with awareness, a player may become aware of her own unawareness and, as a result, switch strategies. In the definition of sequential equilibrium in standard games, play off the equilibrium path is dealt with by viewing it as the limit of "small mistakes" (i.e., small deviations from the equilibrium strategy). Given that there are alternative ways of dealing with mistakes in games with awareness, perhaps other approaches for dealing with offequilibrium play might be more appropriate. While other ways of dealing with mistakes may well prove interesting, we would argue that our generalization of sequential equilibrium can be motivated the same way as in standard games. Roughly speaking, for us, how a players awareness level changes over time is not part of the equilibrium concept, but is given as part of the description of the game.

More generally, we have focused here on generalizing solution concepts that have proved useful in standard games, where there is no lack of awareness. The discussion above suggests that introducing awareness allows us to consider other solution concepts. For example, Ozbay [2006] proposes an approach where a player's beliefs about the probability of revealed moves of nature, that the player was initially unaware of, are formed as part of the equilibrium definition. We hope to explore the issue of which solution concepts are most appropriate in games with awareness in future work.

\section{Acknowledgments}

We thank Yossi Feinberg and Aviad Heifetz for useful discussions on awareness. This work was supported in part by NSF under grants CTC-0208535, ITR-0325453, and IIS-0534064, by ONR under grants N00014-00-1-03-41 and N00014-01-10-511, and by the DoD Multidisciplinary University Research Initiative (MURI) program administered by the ONR under grant N00014-01-1-0795. Some of this work was done while the first author was at the School of Electrical and Computer Engineering at Cornell University, U.S.A., supported in part by a scholarship from the Brazilian Government through the Conselho Nacional de Desenvolvimento Científico e Tecnológico (CNPq).

\section{References}

Battigalli, P. (1997). On rationalizability in extensive games. Journal of Economic Theory 74, 40-61.

Bernheim, B. D. (1984). Rationalizable strategic behavior. Econometrica 52(4), 1007-1028.

Feinberg, Y. (2004). Subjective reasoning_games with unawareness. Technical Report Resarch Paper Series \#1875, Stanford Graduate School of Business.

Feinberg, Y. (2005). Games with incomplete awareness. Technical Report Resarch Paper Series \#1894, Stanford Graduate School of Business.

Halpern, J. Y. and L. C. Rêgo (2006). Extensive games with possibly unaware players. In Proc. Fifth International Joint Conference on Autonomous Agents and Multiagent Systems, pp. 744-751. Full version available at arxiv.org/abs/0704.2014.

Kreps, D. M. and R. B. Wilson (1982). Sequential equilibria. Econometrica 50, 863-894.

Li, J. (2006a). Dynamic games with unawareness. Unpublished manuscript.

Li, J. (2006b). Information structures with unawareness. Unpublished manuscript.

Li, J. (2006c). Modeling unawareness without impossible states. Unpublished manuscript.

Myerson, R. (1978). Refinements of the Nash equilibrium concept. International Journal of Game Theory $7,73-80$.

Myerson, R. B. (1991). Game Theory. Cambridge, Mass.: Harvard University Press.

Osborne, M. J. and A. Rubinstein (1994). A Course in Game Theory. Cambridge, Mass.: MIT Press.

Ozbay, E. (2006). Unawareness and strategic announcements in games with uncertainty. Unpublished manuscript.

Pearce, D. G. (1984). Rationalizable strategic behavior and the problem of perfection. Econometrica 52(4), 1029-1050.

Selten, R. (1975). Reexamination of the perfectness concept for equilibrium points in extensive games. International Journal of Game Theory 4, 25-55. 